\title{
Topology of the Deformation of a Non-uniform Grain Structure
}

\author{
Jae-Yong CHAE, Rongshan QIN and H. K. D. H. BHADESHIA \\ Graduate Institute of Ferrous Technology (GIFT), Pohang University of Science and Technology (POSTECH), Pohang 790-784, \\ Republic of Korea.
}

(Received on July 25, 2008; accepted on October 22, 2008)

\begin{abstract}
The study of changes in the amount of grain boundary surface (or edge) as a function of plastic strain is important in the design of metallic materials. The quantitative expression of these changes is usually made assuming idealised and uniform grain structures. In this work we develop a method for treating a distribution of grain sizes and shapes, and demonstrate that this modification in fact has little effect on the evolution of grain parameters during plastic deformation.
\end{abstract}

KEY WORDS: topology; austenite grain structure; non-uniform grains; rolling; deformation.

\section{Introduction}

Polycrystalline materials such as steel and aluminium are frequently thermomechanically processed to optimise properties and achieve the required shape. Of particular interest is the consequential change in the amount of grain boundary area per unit volume $\left(S_{V}\right)$ and grain edge length per unit volume $\left(L_{V}\right)$, parameters which are important in the mathematical modelling of phase transformations ${ }^{1-8)}$ and recrystallisation processes in general. ${ }^{9-12)}$ Underwood expressed these parameters as a function of the extent of deformation using stereological methods, which have the advantage of avoiding assumptions about grain shape as long as space is filled. ${ }^{13)}$ The method cannot however be adapted to complex combinations of deformations.

Other approaches involve analytical equations or numerical computations based on a variety of approximations of the three-dimensional shape of grains, for example, spheres, cubes and tetrakaidecahedra. ${ }^{14-24)}$ These methods can be adapted to combinations of deformations ${ }^{24)}$ but they assume that all the grains are exactly identical in shape and size. The purpose of the present work was to develop a corresponding model for a non-uniform grain structure. It could be argued that if the normals of all surfaces and all edges are uniformly distributed in space, calculations of the new values after deformation only depend on the initial inclinations of each segment or edge and are unaffected by their neighbours. In such cases, the initial topology should not affect the result. However, as will be seen later, new edges and inclinations are created when a non-uniform grain structure is deformed so it remains relevant to study this problem in detail.

\section{Method}

In previous work the tetrakaidecahedron was represented by a set of vectors parallel to the edges of the shape. ${ }^{20,24)}$ The deformation is then implemented by multiplying each of these vectors by an appropriate deformation matrix; the resultant vectors then define the new surfaces and edges to enable the calculation of $S_{V}$ and $L_{V}$ respectively. Only one tetrakaidecahedron needs to be explicitly treated since all others are identical. This simplification is no longer possible when the grain structure is not uniform. Every single edge in the polycrystal would need to be treated separately, a process which is computationally tedious.

A different approach is therefore adopted, in which a uniform array of identical tetrakaidecahedra is perturbed at all the vertices using a stochastic process to generate a nonuniform grain structure.

Consider a single tetrakaidecahedron grain which has 24 vertices, 8 hexagonal and six square faces, and 36 edges, each of length $a$. Only six vectors are needed to completely describe a single grain. The distance between opposing square faces is designated $L=2 \sqrt{2 a}$, Fig. 1. The orthogonal coordinates $x, y$ and $z$ axes are defined normal to the square faces. Uniformly sized tetrakaidecahedra are then stacked on to a body-centred cubic lattice with lattice parameter $L$ as illustrated in Fig. 1. The volume considered in the calculations that follow has the dimensions $20 L \times 20 L \times 15 L$ with $L=5 \mu \mathrm{m}$, enclosing a total of 12000 grains.

Each surface is then shared by two grains, each edge by three and each vertex by four grains. For this uniform array of grains, the edges are henceforth referred to as the pri-
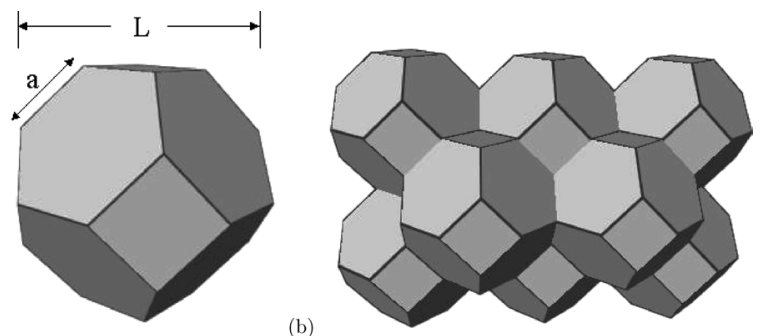

Fig. 1. (a) The Kelvin tetrakaidecahedron. The $x, y$ and $z$ axes are normal to the square faces. (b) Space-filling stack of eight such tetrakaidecahedra of identical size. 


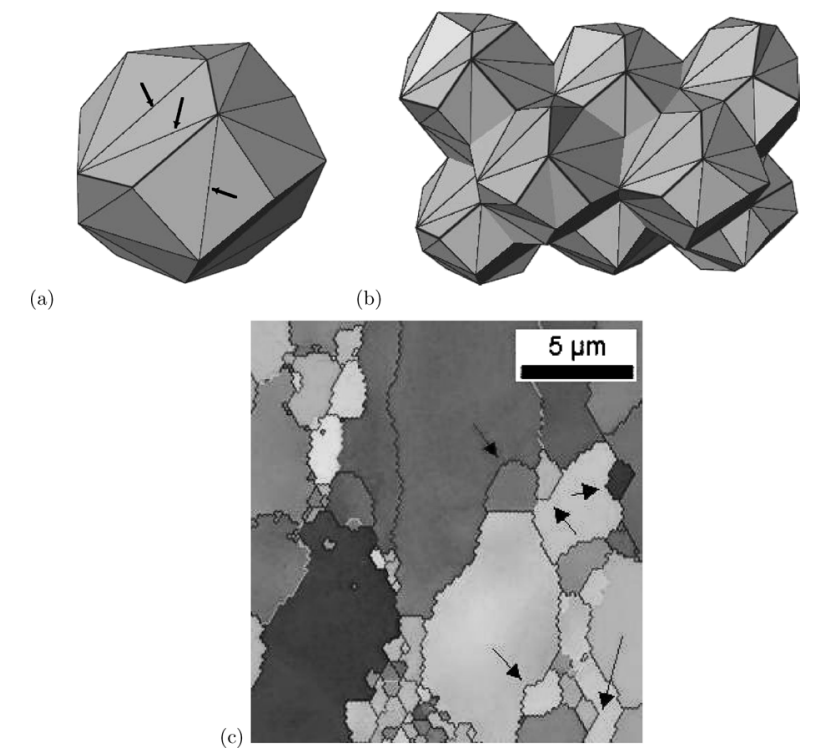

Fig. 2. (a) A non-uniform grain generated using RVM with $w=0.5$. Note the additional secondary edges on the faces of the grain, some of which are identified by the arrows. (b) A space-filling stack of eight non-uniform grains similarly generated. Notice that each grain is different. (c) A crystallographic orientation image of a non-uniform grain structure in a steel, illustrating real secondary edges (courtesy of Ms Lan Huifang, Northeastern University, ShenYang, China).

mary grain edges.

A non-uniform but space-filling grain structure is then generated by a topological transformation in which the vertices are randomly perturbed to new positions. The resulting non-uniform grain still has 24 vertices, a maximum of 44 triangular surfaces and a maximum of 66 edges of various lengths - 66 vectors are therefore needed to describe each grain. The additional edges are henceforth referred to as the secondary and connect just two grains; they occur in real grain structures, appearing as protrusions in two dimensional sections (Fig. 2). Note that the geometry of each planar surface can be any polygon, not simply triangular.

There are two topological transformations created to achieve the non-uniform grain structure. The first is designated the randomised vertex method (RVM), in which the vertex position $r_{0}$ is transformed into the new location $r$ according to

$$
r=r_{0}+\frac{w}{4}(\xi-0.5) L
$$

where $w \leq 1$ is a weight which defines the extent of the fluctuation, and $0<\xi<1$ is a random number.

Figure 2 shows a grain generated using the random perturbations Eq. (1) and the corresponding set of eight grains similarly generated. The grain size distribution itself is naturally affected by such a transformation; Fig. 3 shows how the distribution of grains changes from a uniform grain volume of $62.5 \mu \mathrm{m}^{3}$ as a function of the weight $w$. The method clearly is successful in producing a three-dimensional distribution of grain sizes. The second topological transformation is the rescale randomised vertex method (RRVM) in which the vertex is modified as follows:

$$
\left|r-r_{\text {ref }}\right|=\zeta\left|r_{0}-r_{\text {ref }}\right| \text {. }
$$

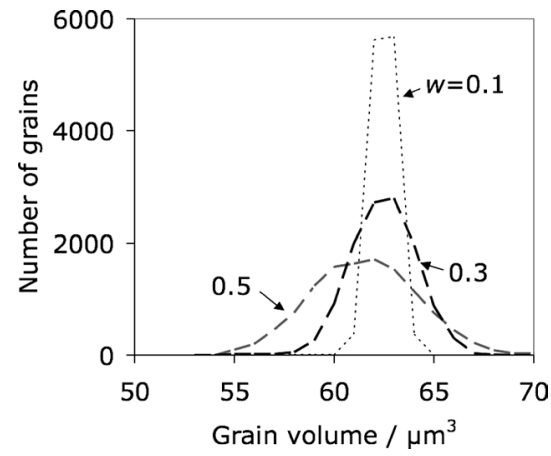

Fig. 3. Grain volume distribution using the RVM and a variety of weights. The total number of grains is 12000 . The number plotted on the vertical axis represents the value for $x \pm 0.5 \mu \mathrm{m}^{3}$

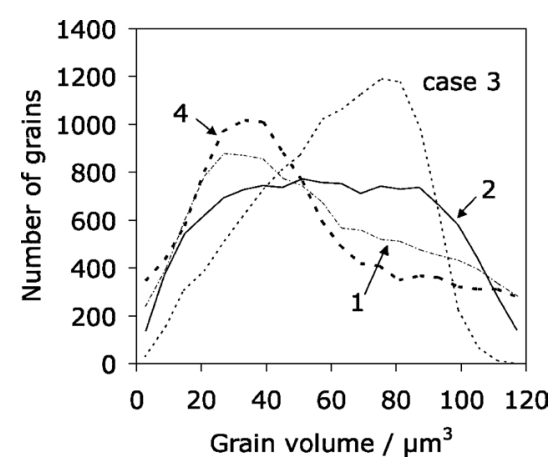

Fig. 4. Grain volume distribution using the RRVM topological transformation for the four cases corresponding to Eq. (3) with $0 \leq \xi \leq 1$. The total number of grains is 12000 . The number plotted on the vertical axis represents the value for $x \pm 3 \mu \mathrm{m}^{3}$.

where $r_{\text {ref }}$ is a reference point inside the grain, $0 \leq \zeta \leq 1$ is a weight factor which can be defined in a number of ways. For the present purpose, four cases are considered:

Case 1: $\zeta=\xi$

Case 2: $\quad \zeta=\sqrt{ } \xi$

Case 2: $\zeta=\xi^{1 / 4}$

Case 2: $\quad \zeta=\exp \left\{-2(\xi-1)^{2}\right\}$

In this, $\zeta$ is a fixed number which is taken from $\xi$ where the latter is equivalent to white noise which is evenly distributed between 0 and 1. The manipulations in Eq. (3) change this into what is known as coloured noise, which makes some values more probable than others. For 12000 grains, $\zeta$ is chosen 12000 times using $\xi$. The outcomes of this process, beginning with a uniform array of tetrakaidecahedra, each of volume $62.5 \mu \mathrm{m}^{3}$, are shown in Fig. 4 . Some two-dimensional sections are illustrated in Fig. 5.

\section{Deformation}

Once the grain structure is defined, it is possible to homogeneously deform it by applying an appropriate mathematical deformation matrix to each vertex. The details have been described thoroughly in a previous paper ${ }^{24)}$ and hence are not repeated here. The matrix representations of a variety of common deformations are also listed there, and it is emphasised that the method can be used for arbitrary homogeneous deformations. We now proceed to compare the changes in grain surface and edge length for uniform ${ }^{24)}$ and 
non-uniform grain structures.

In Fig. 6, $S_{V} / S_{V_{0}}$ represents the amount of grain surface per unit volume normalised by the quantity before deforma-

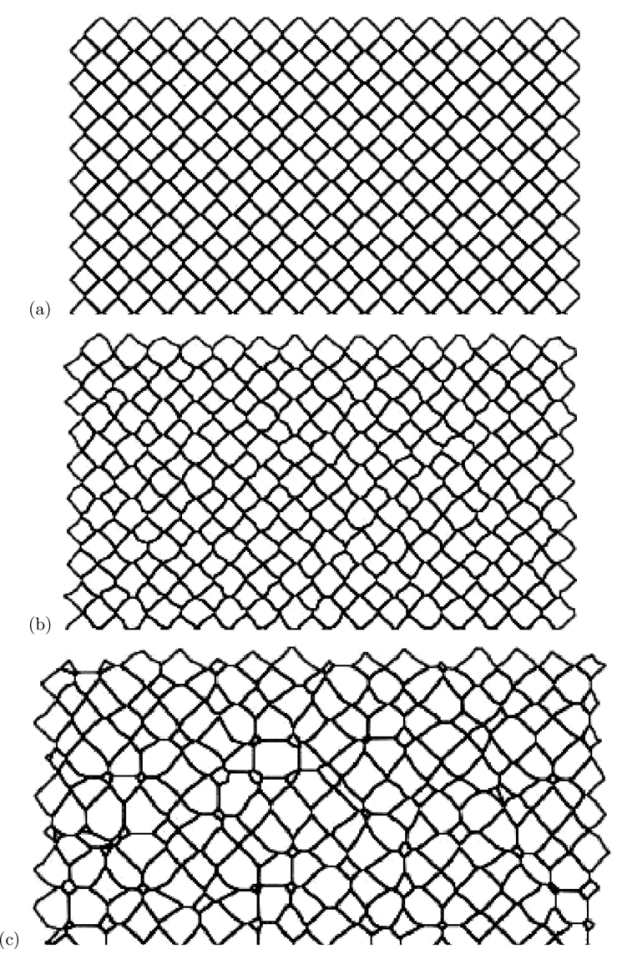

Fig. 5. (a) Section (at $x=57 \mu \mathrm{m}$ ) through a regular stack of uniform tetrakaidecahedra with $L=5 \mu \mathrm{m}$. (b) Same section after the stack was transformed by RVM with $w=0.5$. (c) As (b) but with RRVM Case 2 transformation added.

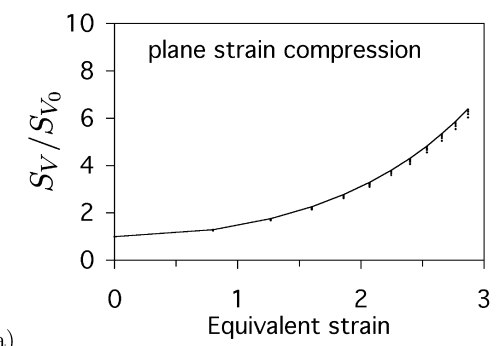

(a)

Equivalent strain tion; $L_{V} / L_{V_{0}}$ is the corresponding ratio for grain edges (including both primary and secondary edges). Calculations are shown for eight kinds of non-uniform grain structures (cases 1-4, with two values of $w$ at 0 and 0.5 ). The individual cases are not distinguished for the sake of clarity given that they produce similar results, all of which reasonably match the curve representing the analytical solution for a uniform grain structure. ${ }^{24)}$ It is evident that the difference brought about by introducing a non-uniform grain structure on the grain surface and edge-length ratios is not significant.

The reason why the results do not change much when the grain structure becomes non-uniform is evident from Fig. 7, where it is seen that $S_{V_{0}}$ and $L_{V_{0}}^{P}$ (primary edge length per unit volume) do not change dramatically when there is a distribution of grain sizes and shapes within the reasonable limits considered here.

It is worth discussing the physical significance of the secondary edges $L_{V_{0}}^{S}$. In our models, the grain faces are geometrically constrained to be flat, for both uniform and nonuniform grain structures. In order to accommodate non-uniformity whilst at the same time fill space, it is necessary during the topological transformations to introduce these secondary edges. As pointed out earlier, these can be real, but many of them could be regarded simply as introducing curvature in the grain faces of non-uniform structures. This would, given the opportunity, drive grain growth.

Figure 8 shows the effects of axisymmetric deformations (e.g., wire drawing, forging) on the surface and edge ratios. The uniformity of grain structure again does not make much of a difference to the results. For the axisymmetric

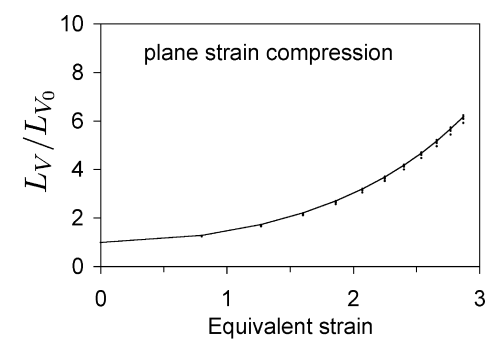

Fig. 6. Calculations for plane strain deformation. (a) $S_{V} / S_{V_{0}}$, (b) $L_{V} / L_{V_{0}}$. The curves represent the analytical solutions for a uniform set of space-filling tetrakaidecahedra. The points are from calculations for a non-uniform set of spacefilling grains. There are eight points per value of strain, representing cases $1-4$ for two values of $w$ at 0 and 0.5 .

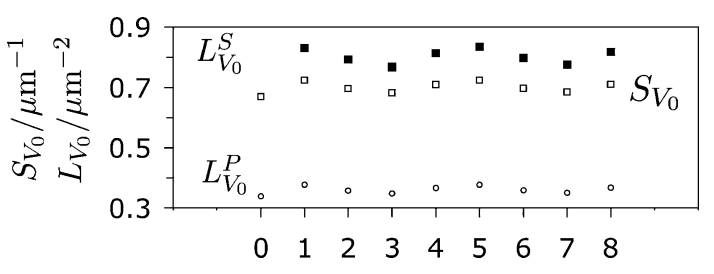

Fig. 7. Parameters describing the undeformed state. The superscripts $P$ and $S$ refer to primary and secondary respectively. On the horizontal axis, 0 represents a uniform grain structure, 1-4 correspond to cases $1-4$ with $w=0$, and 5-8 for cases $1-4$ with $w=0.5$.
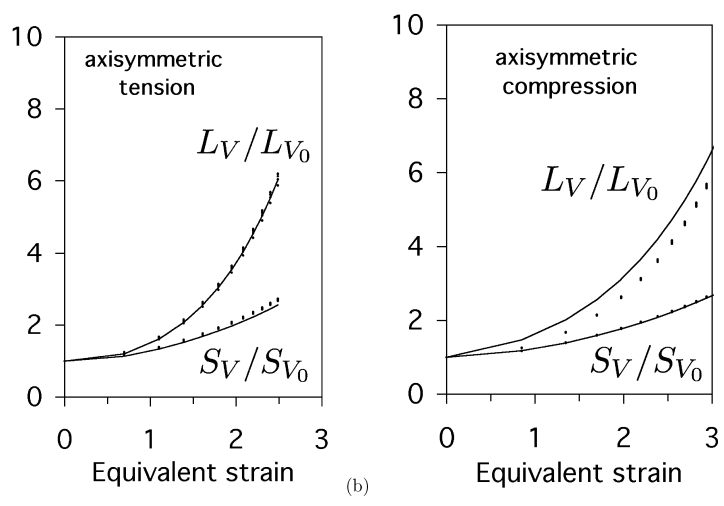

Fig. 8. (a) Axisymmetric tension. (b) Axisymmetric compression. The curves are calculations for uniform grain structures, and the points are data for the eight cases of nonuniform grain structures. 
compression case, the ratio $L_{V} / L_{V_{0}}$ is smaller than expected from uniform grains. This is because the non-uniform grains are less isotropic. Edges can contract as well as expand during compression depending on their orientation relative to the principal axes of the deformation. The effect of orientation is far smaller for a regular tetrakaidecahedron than for an object with large anisotropy ( $c f$. Figs. $2,8^{24)}$ ).

\section{Summary}

We have verified that the same general behaviour occurs as described above for simple shear, cross-rolling and combinations of plane-strain compression and simple shear. In other words, the introduction of non-uniformity in the grain structure does not sufficiently affect the calculated changes in grain surface area and grain edge as a function of deformation.

The computer programs associated with this work are available on www.msm.cam.ac.uk/map/mapmain.html

\section{Acknowledgements}

The authors are grateful to Professor Hae-Geon Lee for the provision of laboratory facilities at GIFT.

\section{REFERENCES}

1) J. W. Cahn: Acta Metall., 4 (1956), 449.

2) M. Umemoto, Z. H. Guo and I. Tamura: Mater. Sci. Technol., 3 (1987), 249.

3) A. Kumar, C. McCulloch, T. B. Hawbolt and I. V. Samarasekera: Mater. Sci. Technol., 7 (1991), 360.

4) E. Annelie: ISIJ Int., 32 (1992), 440.
5) S. Denis, D. Farias and A. Simon: ISIJ Int., 32 (1992), 316.

6) K. J. Lee, J. K. Lee, K. B. Kang and O. Kwon: ISIJ Int., 32 (1992), 326.

7) R. C. Reed and H. K. D. H. Bhadeshia: Mater. Sci. Technol., 8 (1992), 421.

8) S. Jones and H. K. D. H. Bhadeshia: Acta Mater, 45 (1997), 2911.

9) C. M. Sellars: Mater. Sci. Technol., 6 (1990), 1072.

10) C. M. Sellars: Mater. Sci. Technol., 8 (1992), 1090.

11) C. M. Sellars: Ironmaking Steelmaking, 22 (1995), 459.

12) M. P. Black, R. L. Higginson and C. M. Sellars: Mater. Sci. Technol., 17 (2001), 1055.

13) E. E. Underwood: Quantitative Stereology, Addison-Wesley Publication Company, (1970).

14) J. Gil-Sevillano, P. van Houtte and E. Aernoudt: Prog. Mater. Sci., 27 (1980), 69.

15) O. Knustad, H. J. McQueen, N. Ryum and J. K. Solberg: Pract. Metallogr., 22 (1985), 215.

16) H. E. Vatne, T. Furu, R. Orsund and E. Nes: Acta Mater., 44 (1996), 4463.

17) Y. van Leeuwen, S. Vooijs, J. Sietsma and S. V. der Zwaag: Metall. Mater. Trans. A, 29A (1998), 2925.

18) I. Czinege and T. Reti: Determination of local deformation in cold formed products by a measurement of the geometric characteristics of the crystallites: in: Eighteenth International Machine Tool Design and Research Conference, Forming, Vol. 1, (1977), 159.

19) M. Umemoto, H. Ohtsuka and I. Tamura: Trans. Iron Steel Inst. Jpn., 23 (1983), 775.

20) S. B. Singh and H. K. D. H. Bhadeshia: Mater. Sci. Technol., 15 (1998), 832.

21) K. Matsuura and Y. Itoh: Mater. Trans. JIM, 32 (1991), 1042.

22) Y. Takayama, N. Furushiro, T. Tozawa, H. Kato and S. Hori: Mater. Trans. JIM, 32 (1991), 214.

23) P. Bate and W. B. Hutchinson: Scr. Mater, 52 (2005), 199.

24) Q. Zhu, C. M. Sellars and H. K. D. H. Bhadeshia: Mater. Sci. Technol., 23 (2007), 757. 